\title{
Square sum labeling for some lilly related graphs
}

\author{
A. Edward Samuel and S. Kalaivani" \\ Ramanujan Research Centre PG and Research, Department of Mathematics, Government Arts College \\ (Autonomous), Kumbakonam, Tamilnadu, India
}

C2017 ACCENTS

\begin{abstract}
Let $G$ be a graph is said to be a square sum labeling if there exist a bijectionf $: V(G) \rightarrow\{0,1,2, \ldots, p-1\}$ such that the induced function $f^{*}: E(G) \rightarrow N$ given by $f^{*}(u v)=[f(u)]^{2}+[f(v)]^{2}$, for every $u v \in E(G)$ are all distinct. A graph which admits square sum labeling is called a square sum graph. In this paper we investigate square sum labeling for some graphs related to lilly graph. We discuss square sum labeling in the context of some graph operations namely duplication, fusion, switching in the lilly graph $I_{n}$. We prove that the duplication of an apex vertex and other vertex of lilly graph are square sum graph. We prove that the identifying any vertex of lilly graph are square sum graph. We prove that the switching of an apex vertex and other vertex of lilly graph are square sum graph and also apply coloring of the graph operations of the lilly graph is satisfying coloring condition.
\end{abstract}

\section{Keywords}

Square sum labeling, Square sum graph, Lilly graph, Duplication, Fusion, Switching, Coloring.

\section{Introduction}

In this paper, we consider only simple, finite, undirected and non - trivial graph $G=(V(G), E(G))$ with the vertex set $V(G)$ and the edge set $E(G)$. For all other terminology and notations in graph theory I follow West [3]. If the vertices of the graph are assigned values subject to certain conditions is known as graph labeling. A dynamic survey on graph labeling is regularly updated by Gallian [5] and it is published by Electronic Journal of Combinatory. In [9] A. Edward Samuel and S. Kalaivani have proved that prime labeling for some octopus related graphs. In [10] A. Edward Samuel and S. Kalaivani have proved that prime labeling for some planter related graphs. In [11] A. Edward Samuel and S. Kalaivani have proved that prime labeling for some Vanessa related graphs. In [8] Germina K.A and Reena Sebastian have proved that On Square sum graphs. In [4] Ajitha .V, Arumugam .S and Germina .K.A have proved that On Square sum graphs. In [2] Frank Harary have proved some results on Graph theory. Vast amount of literature is available on different types of graph labeling and more than 1000 research papers have been published so far in past three decades.

*Author for correspondence

68
For any labeling problems following three characteristics are really note-worthy : A set of numbers from which vertex labels are chosen; A rule that assigns a value to each edge; A condition that these values must satisfy. The present work is aimed to discuss one such a labeling namely square sum labeling.

\section{Preliminary definitions \\ Definition [7]}

Let $G=(V(G), E(G))$ be a graph is said to be a square sum labeling if there exist a bijection $f$ : $V(G) \rightarrow\{0,1,2, \ldots, p-1\}$ such that the induced function $f^{*}: E(G) \rightarrow N$ given by $f^{*}(u v)=$ $[f(u)]^{2}+[f(v)]^{2}$, for every $u v \in E(G)$ are all distinct. A graph which admits square sum labeling is called a square sum graph.

\section{Definition [6]}

Duplication of a vertex $v_{k}$ of a graph G produces a new graph $\mathrm{G}_{1}$ by adding a vertex $v_{k}{ }^{\prime}$ with $N\left(v_{k}{ }^{\prime}\right)=$ $N\left(v_{k}\right)$. In other words a vertex $v_{k}^{\prime}$ is said to be a duplication of $v_{k}$ if all the vertices which are adjacent to $v_{k}$ are now adjacent to $v_{k}{ }^{\prime}$ also.

\section{Definition [6]}

Let $u$ and $v$ be two distinct vertices of a graph G. A new graph $\mathrm{G}_{1}$ is constructed by identifying(fusing) two vertices $u$ and $v$ by a single vertex $x$ is such that 
every edge which was incident with either $u$ or $v$ in $\mathrm{G}$ is now incident with $x$ in $\mathrm{G}_{1}$.

\section{Definition [6]}

A vertex switching $G_{v}$ of a graph $\mathrm{G}$ is obtained by taking a vertex $v$ of $\mathrm{G}$, removing all the entire edges incident with $v$ and adding edges joining $v$ to every vertex which are not adjacent to $v$ in $\mathrm{G}$.

\section{Definition [1]}

A $k$-coloring of a graph $G=(V, E)$ is a function $c: V \rightarrow C$, where $|c|=k$. (Most often we use $c=[k])$. Vertices of the same color form a color class. A coloring is proper if adjacent vertices have different colors. A graph is $k$-colorable if there is a proper $k$-coloring. The chromatic number $\chi(G)$ of a graph $\mathrm{G}$ is the minimum $k$ such that $\mathrm{G}$ is $k-$ colorable.

\section{Square sum labeling for some lilly related graphs}

3.1Lilly graph

The Lilly graph $I_{n}, n \geq 2$ can be constructed by two star graphs $2 K_{1, n}, n \geq 2$ joining two path graphs $2 P_{n}, n \geq 2$ with sharing a common vertex. i.e., $I_{n}=$ $2 K_{1, n}+2 P_{n}$.

In Figure 1, represents the lilly graph. If $n=4$, i. e. , $I_{4}=2 K_{1,4}+2 P_{4}$.

\section{Example 3.2}

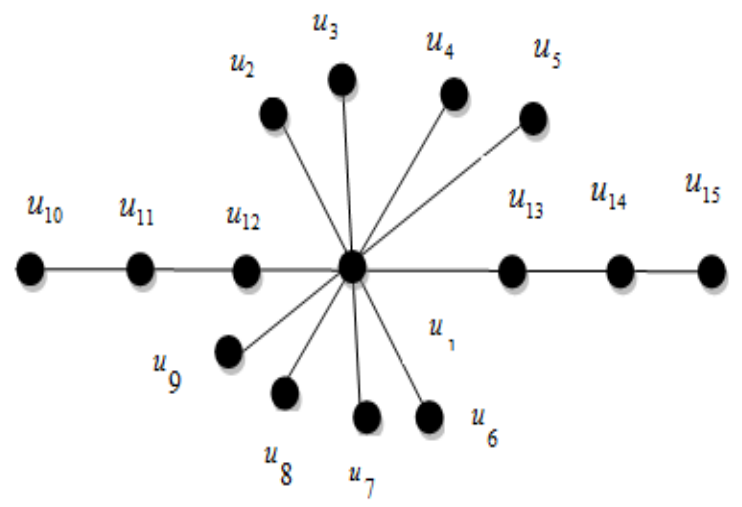

Figure 1 The lilly graph $I_{4}$

Theorem 3.3. The lilly graph $I_{n},(n \geq 2)$ admits square sum labeling, where $n$ is any positive integer.

Proof.Let $G$ be the lilly graph $I_{n},(n \geq 2)$. Let $\left\{u_{1}, u_{2}, \ldots, u_{4 n-1}\right\}$ be the vertices of $I_{n}$. Here $\left|V\left(I_{n}\right)\right|=4 n-1$. Define a labeling $f: V\left(I_{n}\right) \rightarrow$ $\{0,1,2, \ldots, 4 n-2\}$ as follows

69

$$
f\left(u_{i}\right)=i-1 \text { for } 1 \leq i \leq 4 n-1
$$

Clearly vertex labels are distinct. And $f$ induces a function $f^{*}: E(G) \rightarrow N$ given by $f^{*}(u v)=$ $[f(u)]^{2}+[f(v)]^{2}$. Then for any edge $f^{*}\left(e_{i}\right) \neq$ $f^{*}\left(e_{j}\right), i \neq j$. Thus $f$ admits square sum labeling. Therefore $I_{n}$ is a square sum graph.

In Figure 2 represents the lilly graph satisfies the conditions of prime labeling and coloring with respect to the above theorem 3.3 .

\section{Example 3.4}

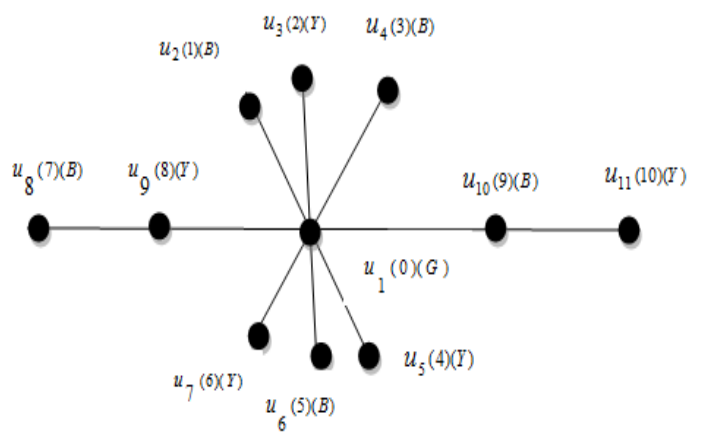

Figure 2 Square Sum labeling for $I_{3}$

Theorem 3.5. The graph obtained by duplication of an apex vertex $u_{1}$ in the lilly graph $I_{n},(n \geq 2)$ is a square sum graph, where $n$ is any positive integer.

Proof.Let $G$ be the lilly graph $I_{n}$. Let $u_{1}$ be an apex vertex of the lilly graph $I_{n}, u_{1}{ }^{\prime}$ be its duplicated of an apex vertex and $G_{k}$ be the graph resulted due to duplication of an apex vertex $u_{1}$ in $I_{n}$, where $n$ is any positive integer. Let $V\left(I_{n}\right)=\left\{u_{1}, u_{2}, \ldots, u_{4 n-1}\right\}$. Here $\left|V\left(G_{k}\right)\right|=4 n$.

We define a labeling $f: V\left(G_{k}\right) \rightarrow\{0,1,2, \ldots, 4 n-$ $1\}$ as follows.

$$
\begin{aligned}
& f\left(u_{i}\right)=i-1 \quad \text { for } 1 \leq i \leq 4 n-1 \\
& f\left(u_{1}{ }^{\prime}\right)=4 n-1
\end{aligned}
$$

Clearly vertex labels are distinct. And $f$ induces a function $f^{*}: E(G) \rightarrow N$ given by $f^{*}(u v)=$ $[f(u)]^{2}+[f(v)]^{2}$. Then for any edge $f^{*}\left(e_{i}\right) \neq$ $f^{*}\left(e_{j}\right), i \neq j$. The weights of all the edges of the graph $\mathrm{G}$ are distinct. Hence $f$ admits square sum labeling. Therefore $I_{n}$ is a square sum graph.

In this Figure 3, represents the duplication of an apex vertex $u_{1}$ in the lilly graph $I_{4},(n \geq 2)$ satisfies the prime labeling and coloring conditions with respect to the above theorem 3.5.

\section{Example 3.6}




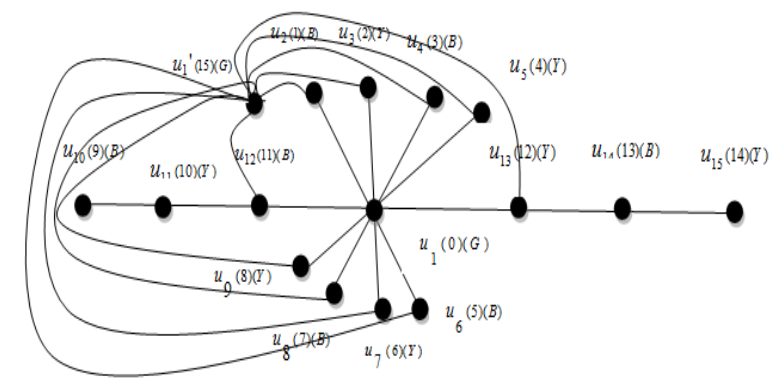

Figure 3 Duplication of an apex vertex $u_{1}$ in $I_{4}$

Theorem 3.7. The graph obtained by duplicating of any vertex $u_{k}$ to $u_{k}{ }^{\prime}$ in the lilly graph $I_{n},(n \geq 2)$ is a square sum graph, where $n$ is any positive integer.

Proof.Let $G$ be the lilly graph $I_{n}$. Let $u_{k}$ be any vertex of the lilly graph $I_{n}, u_{k}{ }^{\prime}$ be its duplicated of any vertex and $G_{k}$ be the graph resulted due to duplication of any vertex $u_{k}$ in $I_{n}$, where $n$ is any positive integer. Let $V\left(I_{n}\right)=\left\{u_{1}, u_{2}, \ldots, u_{4 n-1}\right\}$. Here $\left|V\left(G_{k}\right)\right|=4 n$. The mapping $f: V\left(G_{k}\right) \rightarrow$ $\{0,1,2, \ldots, 4 n-1\}$ by

$$
\begin{aligned}
& f\left(u_{i}\right)=i-1 \quad \text { for } 1 \leq i \leq 4 n-1 \\
& f\left(u_{i}{ }^{\prime}\right)=4 n-1
\end{aligned}
$$

Clearly vertex labels are distinct. And $f$ induces a function $f^{*}: E(G) \rightarrow N$ given by $f^{*}(u v)=$ $[f(u)]^{2}+[f(v)]^{2}$. Then for any edge $f^{*}\left(e_{i}\right) \neq$ $f^{*}\left(e_{j}\right), i \neq j$. The weights of all the edges of the graph $\mathrm{G}$ are distinct. Hence $f$ admits square sum labeling. Therefore $I_{n}$ is a square sum graph.

In this Figure 4, represents the duplication of any vertex $u_{11}$ in the lilly graph $I_{4},(n \geq 2)$ satisfies the prime labeling and coloring conditions with respect to the above theorem 3.7.

\section{Example 3.8}

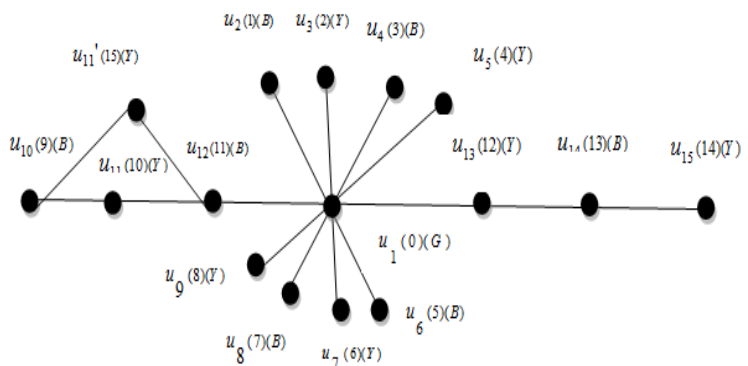

Figure 4 Duplication of any vertex $u_{11}$ in $I_{4}$

Theorem 3.9. The graph obtained by identifying any two vertices $u_{i}$ and $u_{k}$ (where $d\left(u_{i}, u_{k}\right) \geq 3$ ) of the lilly graph $I_{n},(n \geq 2)$ is a square sum graph, where $n$ is any positive integer.
Proof. Let $I_{n},(n \geq 2)$ be the lilly graph with vertices $\left\{u_{1}, u_{2}, \ldots, u_{4 n-1}\right\}$ and the vertex $u_{i}$ be fused with $u_{k}$. Denote the resultant graph as $G_{k}$. Here we note that $\left|V\left(G_{k}\right)\right|=4 n-2$.The mapping $f: V\left(G_{k}\right) \rightarrow$ $\{0,1,2, \ldots, 4 n-3\}$ is defined by

$$
\begin{array}{cl}
f\left(u_{1}=u_{16}\right)=i-1 & \text { for } i=1 \\
f\left(u_{i}\right)=i-1 & \text { for } 2 \leq i \leq 4 n-5 \\
f\left(u_{i}\right)=i & \text { for } 4 n-3 \leq i \leq 4 n-1
\end{array}
$$

Clearly vertex labels are distinct. Then $f$ induces a function $f^{*}: E(G) \rightarrow N$ given by $f^{*}(u v)=$ $[f(u)]^{2}+[f(v)]^{2}$ and for any edge $f^{*}\left(e_{i}\right) \neq f^{*}\left(e_{j}\right)$, $i \neq j$. The weights of all the edges of the graph $\mathrm{G}$ are distinct. Hence $f$ admits square sum labeling. Therefore $I_{n}$ is a square sum graph.

In this Figure 5, represents the identifying of any two vertices $u_{1}$ and $u_{16}$ in the lilly graph $I_{5},(n \geq 2)$ satisfies the prime labeling and coloring conditions with respect to the above theorem 3.9.

Example 3.10

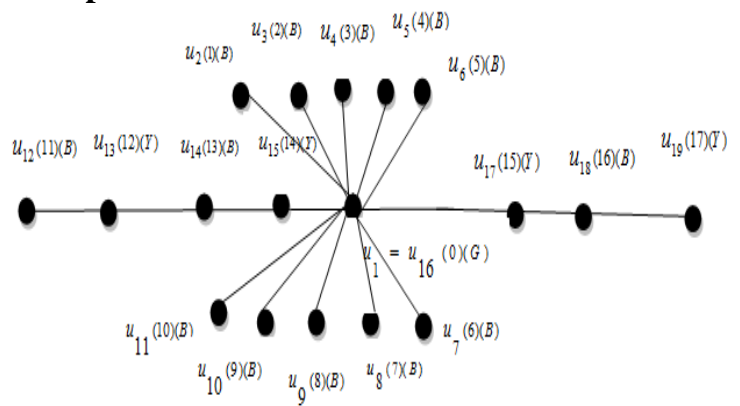

Figure 5 Fusion of $u_{1}$ and $u_{16}$ in $I_{5}$.

Theorem 3.11. The switching of any vertex $u_{k}$ in a lilly graph $I_{n},(n \geq 2)$ produces a square sum graph, where $n$ is any positive integer.

Proof.Let $G=I_{n}$ and $u_{1}, u_{2}, \ldots, u_{4 n-1}$ be the successive vertices of lilly graph $I_{n},(n \geq 2)$ and $G_{u}$ denotes the graph obtained by a vertex switching of $\mathrm{G}$ with respect to the vertex $u$. It is obvious that $\left|V\left(G_{u}\right)\right|=4 n-1$. Without loss of generality we initiate the labeling from $u_{1}$ and proceed in the clockwise direction. Define a labeling $f: V\left(G_{u}\right) \rightarrow$ $\{0,1,2, \ldots, 4 n-2\}$ as

$$
f\left(u_{i}\right)=i-1 \quad \text { for } 1 \leq i \leq 4 n-1
$$

Clearly vertex labels are distinct. Then $f$ induces a function $f^{*}: E(G) \rightarrow N$ given by $f^{*}(u v)=$ $[f(u)]^{2}+[f(v)]^{2}$ and for any edge $f^{*}\left(e_{i}\right) \neq f^{*}\left(e_{j}\right)$, $i \neq j$. The weights of all the edges of the graph $\mathrm{G}$ are distinct. Hence $f$ admits square sum labeling. Therefore $I_{n}$ is a square sum graph. 
In this Figure 6, represents the switching of any vertex $u_{2}$ in the lilly graph $I_{2},(n \geq 2)$ satisfies the prime labeling and coloring conditions with respect to the above theorem 3.11 .

\section{Example 3.12}

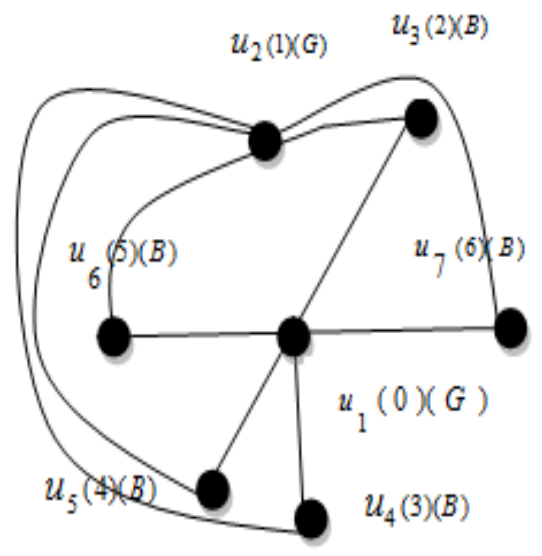

Figure 6 Switching any vertex $u_{2}$ in $I_{2}$

Theorem 3.13. The switching of an apex vertex $u_{1}$ in a lilly graph $I_{n},(n \geq 2)$ produces a square sum graph, where $n$ is any positive integer.

Proof.Let $G=I_{n}$ and $u_{1}, u_{2}, \ldots, u_{4 n-1}$ be the successive vertices of lilly graph $I_{n},(n \geq 2)$ and $G_{u}$ denotes the graph obtained by an apex vertex switching of $\mathrm{G}$ with respect to the vertex $u_{1}$. It is obvious that $\left|V\left(G_{u}\right)\right|=4 n-1$. Without loss of generality we initiate the labeling from $u_{1}$ and proceed in the clock-wise direction. Define a labeling $f: V\left(G_{u}\right) \rightarrow\{0,1,2, \ldots, 4 n-2\}$ as

$$
f\left(u_{i}\right)=i-1 \quad \text { for } 1 \leq i \leq 4 n-1
$$

Clearly vertex labels are distinct. Then $f$ induces a function $f^{*}: E(G) \rightarrow N$ given by $f^{*}(u v)=$ $[f(u)]^{2}+[f(v)]^{2}$ and for any edge $f^{*}\left(e_{i}\right) \neq f^{*}\left(e_{j}\right)$, $i \neq j$. The weights of all the edges of the graph $\mathrm{G}$ are distinct. Hence $f$ admits square sum labeling. Therefore $I_{n}$ is a square sum graph and it is a disconnected graph.

In this Figure 7, represents the switching of an apex vertex $u_{1}$ in the lilly graph $I_{2},(n \geq 2)$ satisfies the prime labeling and coloring conditions with respect to the above theorem 3.13 .

\section{Example 3.14}

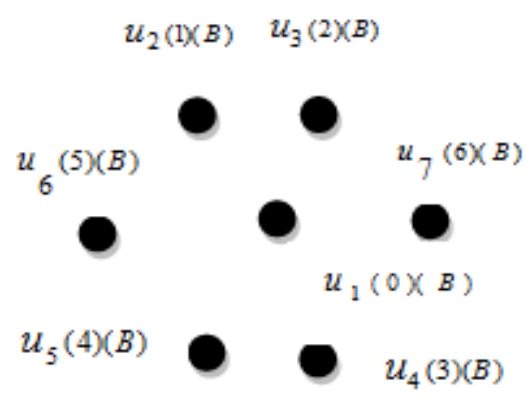

Figure 7 Switching an apex vertex $u_{1}$ in $I_{2}$.

\begin{abstract}
Applications
Several practical problems in real life situations have motivated the study of labeling of graphs which are required to obey a variety of conditions depending on the structure of graphs. Square sum labeling are applied in the additive number theory, coding theory problems, communication network design, X-ray crystallographic analysis, optimal circuit layout, integral voltage generator, telecommunication.
\end{abstract}

\section{Conclusion}

In this paper we proved that the lilly graph $I_{n}$, duplication of the lilly graph $I_{n}$, fusing of the lilly graph $I_{n}$, switching of the lilly graph $I_{n}$ are square sum graphs and also applied coloring in the lilly graph $I_{n}$ are square sum graphs.

\section{Conflicts of interest}

The authors have no conflicts of interest to declare.

\section{References}

[1] Brooks RL. On colouring the nodes of a network. In mathematical proceedings of the cambridge philosophical society 1941 (pp. 194-7). Cambridge University Press.

[2] Harrary F. Graph theory. Narosa Publishing House; 2001.

[3] West DB. Introduction to graph theory. Prentice-Hall, India; 2001.

[4] Ajitha V, Arumugam S, Germina KA. On square sum graphs. AKCE J. Graphs, Combin. 2006; 6(1):1-10.

[5] Gallian JA. A dynamic survey of graph labeling. The Electronic Journal of Combinatorics. 2009; 16(6):1219.

[6] Meena S, Vaithilingam K. Prime labeling for some fan related graphs. International Journal of Engineering Research \& Technology.2012; 1(9):1-19.

[7] Shiama J. Square sum labeling for some middle and total graphs. International Journal of Computer Applications (0975-08887). 2012. 
S. Kalaivani et al.

[8] Germina KA, Sebastian R. On square sum graphs. Proyecciones (Antofagasta). 2013; 32(2):107-17.

[9] Edward Samuel .A and Kalaivani .S. Prime labeling for some octopus related graphs. IOSR Journal of Mathematics.2016; 12(6):57-64.

[10] A. Edward Samuel, S. Kalaivani. Prime labeling for some planter related graphs. International Journal of Mathematics Research. 2016;8(3):221-31

[11] Samuel AE, Kalaivani S. Prime labeling for some vanessa related graphs. Indian Journal of Applied Research. 2017; 7(4).

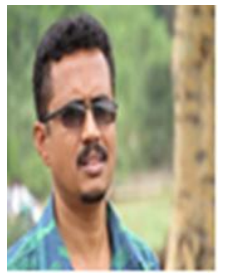

A. Edward Samuel is an Assistant Professor in Department of Mathematics, Government Arts College (Autonomous), Kumbakonam. $\mathrm{He}$ received his Ph.D., in Mathematics from Jamal Mohammed College, Tiruchirapalli. He is the author/coauthor of over 25 research papers with 15 citations including communications in national and international conferences. His research interests are in the areas of fuzzy optimizations and fuzzy graph theory.

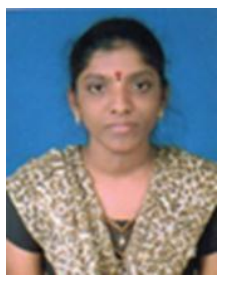

S. Kalaivani is Ph.D., scholar in Department of Mathematics, Government Arts College (Autonomous), Kumbakonam. She is currently working on labeling concepts in graph theory. She is also introduced Vanessa graph, Octopus graph, Planter graph, Lilly graph. She is the author of over 3 research papers were published in international journals.

Email: vanikalai.248@gmail.com 